\title{
Polysèmes
}

Revue d'études intertextuelles et intermédiales

\section{Sépia, couleur de l'encre, teinte du temps}

Sepia, the colour of ink and the colour of time

\section{Jean-Pierre Montier}

\section{(2) OpenEdition}

\section{Journals}

Édition électronique

URL : http://journals.openedition.org/polysemes/570

DOI : $10.4000 /$ polysemes. 570

ISSN : 2496-4212

Éditeur

SAIT

\section{Référence électronique}

Jean-Pierre Montier, "Sépia, couleur de l'encre, teinte du temps », Polysèmes [En ligne], 14 | 2015, mis en ligne le 18 novembre 2015, consulté le 10 décembre 2020. URL : http://journals.openedition.org/ polysemes/570; DOI : https://doi.org/10.4000/polysemes.570

Ce document a été généré automatiquement le 10 décembre 2020.

Polysèmes 


\section{Sépia, couleur de l'encre, teinte du temps}

Sepia, the colour of ink and the colour of time

Jean-Pierre Montier

Je remercie vivement Mmes Anne Cartier-Bresson (conservatrice, Ville de Paris), Danièle Molinari (directrice de la Maison Victor Hugo), Victoria Tebar (Université des Beaux Arts, Barcelone), et Elisabeth Lavezzi (Cellam, Rennes 2) pour les renseignements qu'elles m'ont aimablement fournis.

\section{Une couleur articulant l'ancien et le moderne}

1 Quoi de commun entre les noms d'un quatuor de musique classique québécois, d'un restaurant chic de Bruxelles, d'une maison d'édition consacrée aux voyages, d'une boutique de mode, et d'un appareil photo numérique dernier cri ? Le terme sépia réunit toutes ces activités ou ces objets, assurément hétéroclites; il est toutefois moins nébuleux qu'on ne serait tenté de le penser.

2 À la base de cette ramification sémantique d'apparence composite, il y a deux idées fondues - et historiquement fausses, on le verra - celle de la photographie ancienne et celle d'une teinte chimique, proche de l'ocre, qui aurait été élaborée afin de conserver ces images, perçues contradictoirement comme anciennes et encore présentes. C'est à partir de ce noyau que se déclinent des valeurs telles que le chic, l'art de vivre, le goût de plaire sans ostentation, et un imaginaire social combinant le classicisme avec le souci d'être pleinement de son temps : telles sont les valeurs que l'on trouve dans le quatuor québécois, le restaurant bruxellois, la maison d'édition et la boutique de mode ci-dessus mentionnés. Contrairement aux couleurs des sociétés post-industrielles dont Manlio Brusatin (Brusatin 2009) souligne qu'elles tendent soit à la bichromie noir/ blanc, soit à la profusion du vif, voire du fluo, la sépia en revanche connote synthétiquement un passéisme point bégueule et une modernité point frivole; en somme, un art de (bien) vivre dans la société industrielle. 
3 Aux origines du mot lui-même, il y a un animal, la seiche, que la zoologie classe dans les céphalopodes dibranchiaux. Elle est largement présente dans l'iconographie antique, sur des hanaps, des assiettes, des vases rythons. Dans le monde méditerranéen, le liquide noir dont la seiche ou la pieuvre se servent pour désorienter leurs prédateurs est utilisé à la fois en cuisine (aujourd'hui : en sauce avec des tagliatelles par exemple) et comme encre : en latin impérial, sepia désignait cet animal, ainsi que l'encre qu'il produisait, et sa teinte. Les seiches de l'Adriatique étaient les plus renommées, celles de l'Atlantique engendrant paraît-il des encres moins stables. On en aurait utilisé dans des scriptoriums normands et bretons, car les seiches ont pullulé dans la Manche jusqu'à la Seconde Guerre mondiale. De fait, la fabrication de cette substance est aisée : on fait sécher les poches à encre pour en extraire le jus noir, que l'on déshydrate pour obtenir un objet se présentant sous forme de bâtonnet ou de pain. En termes de chromatisme, il s'agit d'un brun clair un peu fade situé entre le siennois (champ chromatique rouge) et la terre de sienne (champ chromatique brun). Au Moyen Âge, on la conserve diluée dans des flacons de verre, mais on lui préfère généralement des encres d'origine végétale, d'un noir plus intense et moins proche de la couleur du parchemin.

Si l'usage de l'encre brune existe encore de nos jours, en revanche on a le plus souvent oublié son importance passée, et il est encore plus rare qu'on se souvienne de son origine. L'Encyclopédie de Diderot et d'Alembert consacre pourtant le tiers de l'article Seiche à la description de cette encre ${ }^{1}$ :

Lorsqu'on met la seiche hors de l'eau, elle répand une liqueur noire par un petit canal qui aboutit à l'anus; cette liqueur est renfermée dans un sac dont les parois extérieurs sont blancs $[s i c] ;[. .$.$] il contient assez de liqueur pour teindre en noir$ plusieurs seaux d'eau. [...] Si on reçoit cette liqueur dans un vase au sortir du sac, elle se coagule et se durcit en peu de jours; ensuite, elle se gerce et se divise par morceaux qui étant broyés donnent une belle couleur noire. Swammerdam prétend que les Indiens composent l'encre de la Chine avec la liqueur noire de la seiche.

L'encre sépia s'utilisa tant pour l'écriture que le dessin. Un ouvrage de Hobbes datant de 1646, A Minute or First Draught of the Optiques (British Library, Ms. 3360), comporte un dessin montrant la coupe d'un œil, exécuté à la sépia. Il s'agit d'un croquis scientifique, mais des artistes aussi y recouraient, appréciant son brun très particulier, à mi-chemin entre les tons chauds et froids. Elle a été employée comme pigment pour la peinture à l'huile, pure ou avec divers adjuvants (carbonate de potasse et acide), mais c'est dans le lavis qu'on la trouve le plus fréquemment, surtout à partir du milieu du XVIII ${ }^{\mathrm{e}}$ siècle. Ainsi les dessins de Tiepolo (Joseph expliquant les songes, Musée Condé), de Guardi (Assemblée de personnages vénitiens, Musée de Rueil), ou de Greuze (L'Orage, Musée de Tournus). Au début du XIX ${ }^{e}$ siècle, l'usage de la sépia suit deux directions.

D'une part, on a des images destinées à une diffusion relativement rapide, liée au développement de la lithographie, à l'essor du dessin d'actualité. Par exemple, JeanBaptiste Isabey exécute un dessin représentant Napoléon visitant la manufacture de Jouy (1806, Musée de Versailles). Le crayon sépia devient l'instrument du traitement de l'événement restitué presque en temps réel, et ce bien avant que la photographie n'en soit capable. Associé souvent à des papiers de médiocre qualité, dans les journaux, le dessin crayonné est transféré en une gravure qui elle-même jaunit, contribuant à donner à de très nombreuses images cette teinte sépia qui va devenir par la suite la couleur du temps qui fuit (chez les romantiques), ou de la référence au passé (à l'époque moderne). Au début du XIX ${ }^{e}$ siècle, l'on a, sous les auspices de la sépia, une production importante liée à la mise en valeur de ce que l'on appellera le 
"patrimoine ", monumental ou paysager. Vingt ans avant que Hugo n'écrive son fameux Guerre aux démolisseurs, le comte Turpin de Crissé en 1810 représente le Glacier des Bossons près de Chambéry (Musée de Malmaison), à la mine de plomb rehaussée de sépia, qui paraît anticiper à la fois la Mission héliographique recensant le patrimoine architectural français, et les photographies de montagnes des frères Bisson ${ }^{2}$.

D'autre part, c'est une couleur que les artistes romantiques redécouvrent, ou plus exactement à laquelle ils donnent une valeur inédite. Au siècle des Lumières, il y eut bien, à partir de 1751 précisément, une éphémère mode du brun-jaune appelé « caca dauphin » (Pastoureau 2008, 160), pour fêter la naissance du duc de Bourgogne, petitfils de Louis XV. Mais la nuance "moutarde ", proche de la sépia, fut constamment honnie depuis le Moyen Âge, étant considérée comme la plus laide qu'il se puisse percevoir. Plus généralement, le marron est avec le violet « de nos onze couleurs et demi-couleurs la moins aimée, bien qu'elle foisonne dans la nature, les sols, les végétaux. Elle évoque la saleté, la pauvreté » (Pastoureau \& Simonnet 115). Mais à l'époque romantique, s'opère un basculement : les dégradés monochromes de la sépia sont associés à la mode gothique, au fantastique, à l'occulte, voire au satanique. Ce chromatisme devient celui de la mélancolie, de la folie : par exemple chez Caspar David Friedrich, Fenêtre de l'atelier (Nationalgalerie, Berlin) ou Francisco de Goya, Le Sommeil de la raison engendre des monstres (Musée du Prado). C'est la teinte du spleen face aux temps nouveaux ; les romantiques adoptent cette couleur jusqu'alors mal aimée, au point que la sépia devient associée au romantisme lui-même : Paul Morand, dans Londres, publié en 1933 évoque un «[...] brouillard pareil à une sépia romantique : il répand sur les nuages son marc de café, sur les murs de la suie et de la fumée de bougie, comme faisait Victor Hugo, dans ses dessins » (71).

Baudelaire, dans plusieurs de ses articles des Salons de 1846 et 1855, remarque la profusion de l'emploi de la sépia chez les caricaturistes en particulier, et l'effet singulier de cette couleur qui lui semble plus complexe qu'il ne paraît, et qui restituerait selon lui un sentiment de "monstrueux vraisemblable ", fruit d'un art "à la fois transcendant et naturel ». Baudelaire a probablement raison de souligner l'intrinsèque complexité de la sépia : cette couleur met sous tension des contraires, par une sorte d'aptitude à joindre et activer des données antinomiques sans les neutraliser. Ce phénomène paraît en réalité avoir été perçu en des temps reculés; Isidore de Séville, au IV siècle, dans son Etymologiarum sive originum, rapporte ceci : "Cuius atramento tanta vis est ut lucernae addito Aethiopas videri ablato priori lumine quidam tradant " (livre XII, chapitre VI «De Piscibus », § 47). Autrement dit: « [la seiche] grâce à son encre a une si grande puissance que, si on en ajoute à une lampe, on se prend pour des Ethiopiens - le premier éclairage ayant disparu - aux dires de certains ». Isidore souligne le pouvoir d'ambiguïté de l'encre de seiche mélangée à l'huile de lampe, capable de transformer nos Européens en des Éthiopiens, des Noirs... Il y aurait bien une spécificité, même une valeur philosophique de la sépia: elle n'est pas tant une coloration qu'un masque, un voile : comme l'animal dont elle tire son origine, elle joue de la dialectique de l'apparition/disparition, elle mime et leurre dans l'instant même où elle représente, elle fait vaciller nos repères. Dans La Peinture d'abord, en 1942, André Lhôte la place sous le signe de l'oxymore (une figure de la contradiction), faisant de la sépia « un de ces fragiles procédés, ayant le papier pour support, l'eau pour véhicule, la transparence comme force [...] » (64). La « fragilité » du procédé marque la complexité de ses effets, la difficulté de maîtriser leur vacillement sémantique. L'allusion faite par Lhôte à l'eau (le milieu originel de l'animal), qui serait le "véhicule» de la sépia, 
semble suggérer que, même utilisée avec l'aquarelle, il ne s'agit pas tout à fait d'une couleur comme les autres.

\section{Une couleur absente de l'histoire de l'Art, mais une encre présente chez les écrivains}

9 Tandis que le type de couleur que la sépia évoque est parfaitement identifiable du grand public, le terme est presque totalement absent des Histoires de l'Art. Ainsi, le vocable sépia ne figure-t-il même pas en index de l'ouvrage monumental de John Gage (Gage 2008). Il est sans doute plusieurs raisons à cela. La sépia n'est d'abord associée au nom d'aucun grand peintre (au contraire du vert Véronèse, par exemple), ni à aucune école ou mouvement (si elle est assez bien représentée dans le romantisme, c'est davantage comme effet stylistique que comme signe identitaire revendiqué). Dans le vocabulaire des peintres, la nuance est mal identifiée parmi les bruns, les ocres, les teintes terreuses. C'est du reste moins une couleur de peintre que de dessinateur, et encore les dessins qui l'utilisent sont-ils doublement dévalorisés : ce sont souvent des esquisses préparatoires, donc des brouillons; ils sont d'autre part fréquemment diffusés sous forme de gravures ou de cartes, donc des objets mineurs, et même populaires dans le cas des cartes postales conservées au Musée des Arts et Traditions Populaires. On trouve pourtant cette couleur au XXe siècle par exemple chez Mathurin Méheut, qui peint vers 1920 une Noce bigoudène, associant le lavis d'encre sépia à la gouache et aux rehauts d'or. Mais Méheut justement est en marge des courant picturaux de son époque, et tente de promouvoir une esthétique qui reconnaîtrait une valeur égale à l'art officiel (ou d'avant-garde) et aux arts décoratifs, ou populaires justement.

10 La sépia n'étant pas non plus parmi les couleurs prestigieuses de l'héraldique, aucun imaginaire collectif valorisant n'a été conçu à son propos. Comme teinte discriminante, elle est usitée par les philatélistes, ce qui renvoie encore à des pratiques culturelles mineures, spécialisées et confidentielles. Utilisée avec l'aquarelle ou le lavis, elle devient l'adjuvant d'autres procédés, sous lesquels elle disparaît. La base Joconde du Ministère de la Culture précise dans son index terminologique : «Conformément à une décision internationale, les termes de sépia, de bistre et d'encre de chine sont abandonnés au profit des couleurs d'encre (rouge, brun, noir) $»^{3}$. Aussi bien, comme on le verra plus loin à propos des dessins de Victor Hugo, les notices muséographiques des catalogues d'expositions effacent-elles le plus souvent la présence de la sépia sous la mention générique «lavis d'encre brune ", sans qu'il soit possible, sauf à recourir à des analyses chimiques, de savoir s'il s'agit à l'origine d'encre à base de noir de fumée, ferro-gallique ou autre.

11 N'étant pas une véritable couleur de peintre, n'étant qu'une technique marginale du dessin, la sépia est davantage associée au calame ou à la plume qu'au pinceau ou au crayon : en quoi ce serait plutôt une couleur d'écrivain, si les écrivains en avaient, ou si l'on s'y intéressait. Or, ils en ont : qu'on songe par exemple au papier bleu sur lequel écrivait Colette. Parfois, ils fabriquent leur encre: «Je n'en avais pas une goutte et comme il était trop tard pour en demander dans la maison, j'en ai fait avec de l'indigo et de la sépia en tablettes ", écrit George Sand, dans une lettre à Casimir Dudevant, en 1824. Mais notre culture s'est employée à dissocier plume et pinceau, au contraire de la tradition chinoise, comme l'illustrent les ouvrages de François Cheng ${ }^{4}$. 
12 Étant moins une couleur de la palette que de l'écritoire ou du scriptorium, au moins la sépia aurait-elle pu être présente dans l'histoire du livre? Mais c'est le contraire qui s'est produit. Comme le parchemin était souvent ocre, l'on fit tout pour que le papier fût nettement blanc et que symétriquement l'encre s'opacifiât. L'on s'ingénia à la faire dense, l'on voulut qu'elle séchât vite, que sa teinte durât longtemps. Les plus beaux noirs venaient de bois de vigne calcinée ou d'ivoire carbonisé, mélangés à de l'eau ou du vin, avec pour liant de la gomme arabique, du miel, de l'huile, du blanc d'œuf. Alors que l'encre des manuscrits était beige ou brune, soit à cause de l'œuvre du temps soit en raison de modes de fabrication quelque peu aléatoires, s'impose avec l'imprimerie le schème esthétique du «noir sur blanc ", au point que «plus tard les ouvrages imprimés avec des encres d'autres couleurs ou sur des papiers colorés ne ressembleront jamais à de vrais livres » (Pastoureau 2008, 116). Les encres médiévales restaient des lavis qui ne pénétraient pas les fibres du support et ne provoquaient avec lui aucune réaction chimique, mais à l'ère de Gutenberg, l'huile de lin et le sulfate de cuivre donnent une encre visqueuse qui adhère au papier et est d'un noir dense, tout le contraire en somme des incertitudes ou des ambivalences de la sépia.

\section{Couleur photo?}

13 Aussi bien, les historiens des couleurs ne s'y sont-ils que peu intéressés. Elle est à peine évoquée dans le Dictionnaire publié par le CNRS (Mollard-Desfour 175). Elle est mentionnée par Manlio Brusatin dans l'article de l'Encyclopédia universalis, parmi les couleurs de l'âge industriel, mais Pastoureau, qui lui consacre à peine une page et demie dans son Dictionnaire des couleurs de notre temps, en fait remonter l'usage seulement aux lavis de la Renaissance, pour immédiatement la rapprocher de la photographie et du cinéma, et souligner au passage que cette évidence universellement partagée est une erreur :

En fait, ce qui est sépia, ce ne sont pas tant les photographies de ces décennies révolues que l'imaginaire que nous en avons. Le cinéma ne s'y trompe pas, qui, dans les films contemporains, a parfois recours à une telle coloration pour signifier un retour en arrière, situé avant la Première Guerre mondiale, ou même encore dans les années vingt, avant l'invention du cinéma parlant. Or les films produits par ces époques ne montrent aucunement des images sépia, même lorsque la pellicule a viré chimiquement. Les tons sépias ne correspondent donc guère à une réalité ancienne - photographique ou cinématographique - mais bien plutôt à l'image que nous avons, aujourd'hui, de cette réalité. (Pastoureau 2007, 146-147)

Outre le paradoxe relevé plus haut, l'étude de la sépia souffre donc d'un contresens historique, relevé mais nullement élucidé par Pastoureau. L'explication est pourtant assez simple. Si l'usage de la sépia ne fait l'objet, à ma connaissance, d'aucun article, d'aucune recherche de la part des historiens de la photographie ancienne, c'est qu'il ne s'agit pas d'un terme de spécialiste de la photographie. Selon Anne Cartier-Bresson, experte de la restauration des photographies anciennes, le terme de sépia est dénué de valeur scientifique : alors que les multiples inventeurs de procédés de tirage du XIX ${ }^{\mathrm{e}}$ siècle ont rivalisé de sagacité dans la mise au point de formules chimiques pour développer et fixer leurs images sur papier, aucun n'a usé de sépia. Le produit chimique permettant de réaliser des virages sépia est mis sur le marché par la firme Kodak dans les années 1920, c'est-à-dire dans un contexte où la photographie n'est plus depuis longtemps affaire d'ingénieurs ou de bricoleurs de génie, où elle s'est largement 
démocratisée (et Kodak en est le parfait symbole), mais où sa valeur sémiotique et symbolique collective s'est suffisamment affermie, parmi ses utilisateurs et ses destinataires, pour que le public ait souhaité obtenir des images qui fussent en quelque sorte de la couleur de la photographie en soi.

Mais comme d'autres contre-sens, celui-ci repose sur une base véridique. Les procédés à l'albumine, au sulfate de cuivre, etc., donnaient effectivement une gamme de teintes allant du grisâtre à l'ocre, si bien qu'en un sens il était possible de tous les subsumer sous la catégorie générique "sépia", appellation unique permettant de faire l'économie de la mention des multiples techniques. Même les cyanotypes (procédé mis au point dès 1842 par John Herschel), qui comme leur nom l'indique donnaient des teintes bleuâtres, pouvaient à la limite être classés dans cette catégorie, à la condition facilement acceptable que la sépia - dont par ailleurs des peintres soulignaient la proximité d'avec le bleu, à faible dilution - finisse par désigner la couleur interlope de toute photographie ancienne, et plus largement celle de toute image issue de ce procédé, par-delà les variations colorimétriques objectives qu'il était facile de rapporter aux aléas imputables soit à la chimie soit à l'imprimerie. À ces raisons faisant que la sépia est devenue la couleur de la photographie du XIX ${ }^{e}$ siècle, il faudrait probablement en ajouter une autre, tenant cette fois aux sujets photographiés. Outre les portraits, ce sont des paysages historiques et des objets archéologiques que les premiers photographes prennent et surtout publient le plus volontiers. Aussi bien, un nombre considérable de photographies du XIXe siècle a-t-il tout naturellement cette couleur grisâtre ou jaunâtre de la pierre, de la terre ou du sable, dès lors que les sujets représentés sont des Souvenirs et paysages d'Orient, ou les vues d'Un voyageur en Égypte vers 1850, pour reprendre le titre de deux ouvrages de Maxime Du Camp, dont le premier paraît dès 1848.

Ce qui ne signifie pas non plus que la sépia ait été totalement ignorée des praticiens de la photographie. Gustave Le Gray écrit ainsi, en 1850, à propos d'un tirage :

Voici les différentes teintes successives qu'il prend : gris-bleu, teinte neutre, violetbleu, noir-bleu, noir, noir bistré, bistre, sépia colorée, sépia jaunâtre, jaune feuillemorte, gris verdâtre, toujours de plus en plus effacé. Il faut s'arrêter à un de ces tons selon la plus ou moins grande vigueur du cliché. [...] Pour avoir une épreuve de teinte noire, par exemple, après le fixage à l'hyposulfite, il faut que les parties foncées soient au ton sépia et les parties qui doivent former les blancs au gris-bleu en les retirant de dessous le châssis, afin de réparer la perte de ton que donne l'hyposulfite. ${ }^{5}$

Mais comme on peut le constater, Le Gray décline les teintes que prend un papier lors du tirage, et utilise la sépia parmi toute une gamme de couleurs, un peu à la manière dont on use aujourd'hui du cromalin en imprimerie. Ce n'est qu'en 1894 que RouilléLadevèze, d'une famille d'imprimeurs de Tours, correspondant provincial du PhotoClub de Paris, publie un opuscule de 24 pages intitulé Sépia-Photo et Sanguine-Photo ${ }^{6}$. Il ne s'agit toutefois ni de tirage ni de virage, mais d'utiliser « de la couleur en tubes, telle que celle employée par les aquarellistes » et d'étendre "ce mélange avec un large blaireau sur du papier à dessin ordinaire » (6). L'on est clairement dans la lignée des expérimentations des pictorialistes et les suites lointaines de l'esthétique romantique, moins en rapport avec une recherche spécifique à la photographie que dans la logique d'un rapprochement avec le dessin, le titre de l'ouvrage faisant d'ailleurs porter l'accent tant sur la sépia que la sanguine. 
18 Aussi bien, la monochromie de chaque tirage cachant un polychromisme issu des recettes de tirage, lui-même aggravé par les multiples procédés d'imprimerie (héliogravure, similigravure, etc.), la confusion sur la teinte réelle de la photographie ancienne s'est-elle avérée un véritable casse-tête pour les éditeurs contemporains. L' Histoire de la photographie de Lemagny et Rouillé parue en 1986 chez Bordas le résout par un choix purement économique: on y trouve une répartition entre les images reproduites selon des tons plutôt grisâtres que sépia, presque toutes antérieures à 1920, tandis que celles postérieures à cette date sont dans un noir et blanc d'imprimerie standard. Une discrimination qui n'a strictement aucune valeur archéologique : mais il eût été beaucoup plus coûteux de tirer la totalité des pages en quadrichromie et de faire en sorte que toutes les reproductions fussent en des tons fidèles aux originaux. Plus récemment, la Nouvelle Histoire de la photographie dirigée par Frizot (Larousse, 2001) et surtout L'Art de la photographie des origines à nos jours dirigée par André Gunthert et Michel Poivert (Citadelles/Mazenod, 2007) manifestent le souci de restituer à chaque image la teinte de l'original, ou du tirage référent.

Une recherche lexicale dans la littérature du XIX siècle confirme d'ailleurs que la sépia est systématiquement associée à des pastels ou des lavis. Chez Balzac, en 1843 dans Les Illusions perdues: «M. Alexandre de Brebian, le héros de la sépia, le dessinateur qui infestait les chambres de ses amis par des productions saugrenues et gâtait tous les albums du département, accompagnait M. de Bartas ». Au passage, relevons le peu d'estime que Balzac semble porter au procédé; de même chez Jules et Edmond de Goncourt: "Il y a, sur le chevalet de Gavarni, une sépia représentant une grotte des Pyrénées, du temps où il réservait les moindres blancs et les marges» (Journal 1863). Chez Zola, le terme sépia n'est jamais associé à la photographie ; ainsi dans Le Ventre de Paris, en 1873: "Florent souffrait. Il croyait à quelque tentation surhumaine. Il ne voulait plus voir, il regardait Saint-Eustache, posé de biais, comme lavé à la sépia sur le bleu de ciel, avec ses rosaces, ses larges fenêtres cintrées, son clocheton, ses toits d' ardoises ».

Aragon, en 1925, dans Le Paysan de Paris où il est pourtant fait mention abondamment de photographies, n'emploie le terme de sépia que pour référer à une peinture d'Arnold Böcklin (219). La première association explicite entre la photographie et la sépia, sauf erreur, est de 1934, dans Les Célibataires de Montherlant, par un rapprochement elliptique du mot avec «toute l'époque Arago" (celle de Daguerre et Nièpce, donc). Après la Seconde Guerre mondiale, progressivement le cliché se fait jour. Dans un texte publié en 1946 par Francis Ambrière, consacré à la vie des prisonniers de guerre sous l'occupation, et dont le titre est à prendre en antiphrase - Les Grandes Vacances -, on lit (nous soulignons) : « Nous eûmes Pétain rose et or, à cheval ; Pétain tiré en noir, dans le parc de Vichy, en train d'embrasser une fillette ; Pétain en sépia, accoudé à son bureau et prononçant une apostrophe aux prisonniers [...] » (133). C'est en réalité dans les années soixante, lorsque se popularise la photographie en couleur, que par contraste la sépia fleurit : Romain Gary, dans La Promesse de l'aube, en 1960, évoque : «[...] un uniforme de l'armée du Tsar, avec des rubans de décorations rouges, noirs et blancs, des albums de photographies sépia, des cartes postales, des poupées, des chevaux de bois - tout ce petit bric-à-brac que l'humanité laisse derrière elle sur ses rives, à force de couler [...] » (93). Et ce n'est qu'à partir des années 1970 que l'on va trouver une profusion de textes thématisant la photographie et l'anamnèse littéraire sous les auspices de la sépia. Chez Marguerite Yourcenar, en 1974, dans Souvenirs pieux : «Il se trompait, n'ayant jamais, à 
ce qu'il semble, pris la peine de jeter les yeux sur ces clichés vite fanés. Leur ton sépia les empreint d'une inquiétante mélancolie: on les dirait pris sous cette lumière infrarouge à laquelle, assure-t-on, on distingue mieux les fantômes » (938). Chez Perec, dans La Vie mode d'emploi en 1978 : «[...] un petit cliché sépia, représente un jeune capitaine en uniforme de la guerre hispano-américaine avec des yeux sérieux et candides sous des sourcils hauts et fins et une bouche sensible» (558). Chez Claude Simon, dans Les Géorgiques, 1981: "[...] les deux agrandissements photographiques tirés en sépia, représentant, auréolés de sépia artistiquement dégradé, un homme et une femme raides, figés dans des vêtements eux-mêmes raides, figés, lui et elle déjà sans âge " (157). Et dans L'Acacia, en 1989 : «Sur le mur, à la droite du lit dont les draps sont à présent remontés, se trouve un agrandissement photographique sépia encadré d'une large moulure de bois brun » (331).

On voit donc qu'il faut passer par une évolution complexe avant que la sépia ne soit associée par réflexe à la photographie et ne devienne la «couleur du passé " (Pastoureau 2007, 146). Au reste, c'est bien la teinte sépia que Roland Barthes, dans $L a$ Chambre claire, associe à la fameuse révélation de l'image maternelle: «Et je la découvris. La photographie était très ancienne. Cartonnée, les coins mâchés, d'un sépia pâli, elle montrait à peine deux jeunes enfants debout, formant groupe, au bout d'un petit pont de bois dans un Jardin d'Hiver au plafond vitré » (Barthes 1980, 106). Conserver ou espérer, revivre ou conjurer : plutôt que revivre du temps passé, c'est en réalité le métamorphoser que font les photographies, et le fait que ce soit sous les auspices de la sépia est significatif.

\section{La sépia comme « bien commun » de l'écriture et la photographie}

22 Couleur récusée par les historiens de l'art, soupçonnée par les peintres, secrètement adulée par des écrivains, faussement attribuée à l'histoire de la photographie mais unanimement associée à la photographie ancienne ; teinte et procédé archaïque qui fait pourtant aimer notre modernité : la sépia est tout cela. Elle est plus encore. La sépia est à la lisière entre l'écriture et l'image, étant autant du côté de l'encre que de celui du dessin. Dans la photographie, il y a un imaginaire de l'écriture du temps, et c'est la sépia qui le synthétise parfaitement, parce qu'elle est aussi la couleur de l'encre graphologique, de l'écriture littéraire, en tant que cette dernière, d'une autre façon que la photographie, engage elle aussi un imaginaire temporel. Dans ces effets de vacillement qu'on a relevés plus haut, la sépia serait peut-être le symbole de l'acte mimétique, sous ses deux versants, littéraire ou iconique.

Cet acte mimétique, après tout, est bien celui accompli par l'animal qui produit de la sépia, une substance grâce à laquelle la seiche ou la pieuvre leurrent leur prédateur en projetant comme un écran ou une fausse représentation devant lui. C'est je crois chez Victor Hugo qu'on pourra trouver un exemple parfait de fusion entre la pieuvre, l'encre sépia, et l'activité de l'artiste, où la sépia sera au centre d'une opération de métamorphose, de mise sous tension des contraires, selon la logique imaginaire de cette couleur telle que nous l'avons décrite plus haut.

On sait que Victor Hugo utilisait de la sépia, soit pour son encre, soit pour dessiner. Mais chez lui dessiner est l'autre face d'écrire. L'une des premières expositions 
consacrée à ses dessins hors de France eut lieu aux États-Unis ; le $1^{\text {er }}$ juillet 1902, le New York Times titrait: Victor Hugo the artist (exhibition of Sepia Drawings, Portraits and Caricatures of the Poet at the Lenox Library). Des dessins à la sépia; et en effet, le 5 février 1856, de son exil à Guernesey Hugo sollicitait Paul Meurice :

Voudriez-vous ensuite passer chez Susse et y acheter pour moi une douzaine de pains de sépia anglaise (qui, étant anglaise ne se trouve pas en Angleterre) une douzaine de gros crayons noirs dits crayons sauce, et enfin quatre flacons de collodion à un ou deux francs?

La maison Susse, fondée en 1758, se trouvait Passage des Panoramas, à Paris. Elle avait été lancée auprès des artistes romantiques par le sculpteur James Pradier, père de la fille de Juliette Drouet, dont on sait les liens avec Hugo. Quelques années plus tard, le 29 avril 1860, dans une lettre à Baudelaire il écrit :

Vous m'avez envoyé, cher poète, une bien belle page ; je suis tout heureux et très fier de ce que vous voulez bien penser des choses que j'appelle mes dessins à la plume. J'ai fini par y mêler du crayon, du fusain, de la sépia, du charbon, de la suie et toutes sortes de mixtures bizarres qui arrivent à rendre à peu près ce que j'ai dans l'œil et surtout dans l'esprit. Cela m'amuse entre deux strophes.

Sans insister sur la modestie feinte, soulignons que Victor Hugo désamorce l'hostilité de Baudelaire face à la bizarrerie, au caractère " gothique » de son esthétique. Pour ses dessins, il fait feu de tout bois, sépia, charbon, suie, et encore ne mentionne-t-il pas le marc de café ni les taches d'encre! Si l'on veut reconstituer la genèse de ses dessins, il faut procéder à des analyses chimiques, que les musées souvent rechignent à pratiquer dès lors qu'il est nécessaire de prélever un fragment sur les œuvres. La seule qui ait été faite par le Louvre, selon Victoria Tébar (Tébar 2004), concerne un Mirador, dont on a pu découvrir qu'il avait été réalisé avec de l'encre métallogallique.

On ne sait si c'est avec l'un de ses pains de sépia qu'il fit la pieuvre insérée dans son manuscrit personnel des Travailleurs de la mer qu'il demanda à Turner de relier en mai 1866. Ce dessin célèbre est fréquemment utilisé dans les éditions modernes du roman ${ }^{7}$. Les notices adoptent la prudence qui est de mise: «lavis d'encre brune». Mais ce dessin célèbre cache plusieurs énigmes. La première est l'absence de toute mention de l'encre dans la description que le roman fait des armes de la pieuvre, alors que l'énumération en est fort longue. Le mot sépia est lui-même absolument absent d'un texte foisonnant d'érudition, alors qu'Hugo sait absolument tout d'un animal dont il se plaît à montrer combien il concentre la Création, selon le terme savant qui le désigne : sépia microcosmos. Animal araignée, animal microcosme, animal énigme comme Dieu même : c'en est sans doute trop; de la pieuvre, pas un mot sur son encre! Mais il l'intègre à son propre face-à-face d'écrivain-dessinateur avec la Création, l'infini de la Mer, et par-delà avec Dieu.

Ce mystère, l'absence de toute mention de la sépia dans la description de la pieuvre, renvoie à un autre : le dessin est crypté, avec le monogramme $\mathrm{VH}$, ainsi que le signe de l'infini, que dessinent les tentacules. En leur centre, la tête de l'animal est circulaire ; parfaite? Molle et blanchâtre comme un cerveau, elle figure le Mal en tant qu'il n'est pas un accident de la Création, mais est pernicieux, intelligent. Quel autre de ses organes la rapproche-t-il de l'homme? Le seul par lequel elle soit vulnérable : son œil. Organe également circulaire, mou, accolé au cerveau: orbe sur orbe, redondance du Mal. Plastiquement, la pieuvre est deux fois œil ; comme la vision est double chez Hugo : regard qui glace de remords et aptitude visionnaire. La pieuvre est l'alter ego caché de l'écrivain : Hugo, en signant de ses initiales le dessin des tentacules, manifeste qu'il est la pieuvre. Que son activité d'homme de l'encre est identique à celle de la 
pieuvre jetant son liquide sépia dans l'océan. Mais aussi, qu'est-ce qu'un écrivain ? L'homme du songe, confronté au mystère... Ce lavis d'encre ne se donne pas comme la transposition d'un regard sur du réel : consistant non à voir mais à contempler, à songer, l'acte mimétique posé par ce lavis qui fut peut-être fait de sépia instaure un mouvement de traversée des apparences, auquel l'effet du dilué donne consistance (Montier 2006, 27). Comme la photographie, que Victor Hugo avait vu ses fils pratiquer dans l'atelier de Jersey et qui le fascinait - ne commande-t-il pas du collodion en même temps que des crayons et des pains de sépia à Paul Meurice? -, ce dessin au lavis d'encre - dont finalement il importe assez peu qu'il soit ou non réalisé avec de la sépia, tant il en concentre tous les effets, toute la philosophie -, ce dessin ressortit bien de cette logique de la révélation, de l'apparition, de la mise en tension qui nous a servi de fil conducteur. La sépia serait donc devenue la couleur de la photographie ancienne, et réciproquement la photographie du temps passé se serait fondue en la teinte sépia, grâce à cette capacité métamorphique qu'on voit Hugo mettre en œuvre, de sorte que le Temps et la Création nous soient restitués, par elle, à la fois comme des évidences et des énigmes.

\section{BIBLIOGRAPHIE}

Ambrière, Francis. Les Grandes Vacances. Paris : La Nouvelle France, 1946.

Aragon, Louis. Le Paysan de Paris (1926). Paris : Gallimard, « Folio », 1986.

Barthes, Roland. La Chambre claire. Note sur la photographie. Paris : Seuil, 1980.

Brusatin, Manlio. Histoire des couleurs. Paris : Flammarion, « Champs Arts », 2009.

Frizot, Michel (dir.). Nouvelle Histoire de la photographie. Paris : Larousse, 2001.

Gage, John. Couleur et culture. Usages et significations de la couleur de l'Antiquité à l'abstraction. Paris : Thames \& Hudson, 2008.

Gary, Romain. La Promesse de l'aube (1960). Paris : Gallimard, 1996.

Gunthert, André et Michel Poivert (dir.). L'Art de la photographie des origines à nos jours. Paris : Citadelles et Mazenod, 2007.

Lhôte, André. La Peinture d'abord. Paris : Denoël, 1942.

Mollard-Desfour, Annie. Dictionnaire des mots et expressions de couleur. Le noir. Paris : CNRS éditions, 2005.

Montherlant, Henri de. Les Célibataires (1934). Paris : Gallimard, 1962.

Montier, Jean-Pierre. « Texte et dessins dans Les Travailleurs de la mer ». French Studies 60.1

(janvier 2006) : 15-31.

Morand, Paul. Londres. Paris : Plon, 1933.

Pastoureau, Michel et Dominique Simonnet. Le Petit Livre des couleurs. Paris : Seuil, « Points Histoire », 2005. 
Pastoureau, Michel. Dictionnaire des couleurs de notre temps. Paris : Christine Bonneton, 2007.

Pastoureau, Michel. Noir. Histoire d'une couleur. Paris : Seuil, 2008.

Perec, Georges. La Vie mode d'emploi (1978). Paris : Le Livre de Poche, 1995.

Rouillé-Ladevèze, A. Sépia-Photo et Sanguine-Photo. Nabu-Press, 2010.

Simon, Claude. Les Géorgiques (1981). Paris : Éditions de Minuit, 1992.

Simon, Claude. L'Acacia. Paris : Éditions de Minuit, 1989.

Tébar, Victoria. « La création artistique hugolienne, ses apports dans le domaine des procédés techniques » (2004) :

http://groupugo.div.jussieu.fr/groupugo/04-01-24Tebar.htm et

http://groupugo.div.jussieu.fr/groupugo/doc/04-01-24Tebar_Images.pdf.

Yourcenar, Marguerite. Souvenirs pieux. Paris : Gallimard, 1974.

\section{NOTES}

1. Pour procéder aux recherches lexicales indispensables à la rédaction de cet article, nous avons recouru à la base de données Frantext, développée par l'équipe Atilf de Nancy, CNRS/Université de Lorraine (www.atilf.fr). Si les renseignements qui s'y trouvent sont très précieux, en revanche les éditions et naturellement les pages y sont rarement mentionnées. Aussi, pour les références qui suivront, ne serons-nous pas en mesure de fournir ces indications, sauf exception.

2. Les dessins et tableaux cités sont accessibles sur la base Joconde du Ministère français de la Culture.

3. Voir

http://www.culture.gouv.fr/documentation/joconde/fr/partenaires/AIDEMUSEES/

vocabulaires.htm

4. Voir en particulier François Cheng, Vide et plein, Paris : Le Seuil, « Essais », 1991.

5. Gustave Le Gray, Traité pratique de photographie sur papier et sur verre, Paris, 1850, consulté sur la base frantext.fr

6. A. Rouillé-Ladevèze, Sépia-Photo et Sanguine-Photo, Paris : Gauthier-Villars et fils, «Bibliothèque photographique », 1894.

7. Voir par exemple l'édition Eigeldinger chez Garnier-Flammarion, 1980. Le dessin est présenté sur le site de la BnF :

http://expositions.bnf.fr/lamer/cabinet/anthologie/bibliotheque/24.htm

\section{RÉSUMÉS}

Parmi toutes les teintes que nous dénommons le plus ordinairement ou identifions le plus aisément, la couleur sépia semble être l'une de celles, avec les couleurs fondamentales, à laquelle nous reconnaissons volontiers une valeur simple : c'est la couleur de la photographie ancienne, et par métonymie, celle du temps passé. Bien qu'assez universellement partagée, cette représentation est partiellement fausse et recouvre assurément une réalité plus complexe. On 
pourra même se demander si l'apparente monovalence de cette couleur ou cette teinte ne nous permet pas de mettre à jour certains aspects de l'ambiguité fondamentale de l'opération de mimésis, en particulier sous l'angle de la restitution du temps.

Like basic colours, sepia may be among the most easily identified and clearly named colours, being the brown shade of old photographs and, as such, metonymically, the colour of time past. Although widely shared, that view is only partly correct as things are far more complex and sepia cannot be associated with one single value. Beyond its apparent simplicity, sepia might reflect the inherent ambiguity of the notion of mimesis, examined more specifically through the representation of the past and ultimately through the representation of time.

\section{INDEX}

Mots-clés : couleur, sépia, photographie, encre

Keywords : colour, sepia, photography, ink

\section{AUTEURS}

\section{JEAN-PIERRE MONTIER}

Jean-Pierre Montier est Professeur à l'Université Rennes 2. Il a dirigé le Cellam (Centre d'études des littératures et langues anciennes et modernes) de 2003 à 2013, et ses recherches portent sur les relations entre littérature et photographie, via notamment le site http://www.phlit.org qu'il a créé et qu'il anime grâce à une équipe de chercheurs et de doctorants. Il a publié notamment Revoir Cartier-Bresson (codirection Anne Cartier-Bresson, Textuel, 2009), Carrefour Stieglitz (codirection Jay Bochner, PUR, 2012) et Transactions photolittéraires (PUR, 2015); il prépare la parution de Les écrivains vus par la photographie. Il a bénéficié d'une bourse du France-Berkeley Fund en 2015 pour la promotion des recherches en photolittérature. 